\title{
Usages et perceptions des enseignants lors de l'utilisation de la tablette en contexte scolaire
}

\section{Aurélien Fiévez \\ Université de Montréal \\ Thierry Karsenti \\ Université de Montréal}

\author{
Uses and perceptions of teachers \\ using tablet's in educational context.
}

$\mathrm{R}$

ésumé

L'utilisation des tablettes en contexte scolaire est de plus en plus visible dans les salles de classe en Amérique du Nord et en Europe.

L'intégration de cette nouvelle technologie induit des modifications dans les activités d'enseignement et d'apprentissage. La tablette apporte de multiples perspectives et de nouveaux usages pédagogiques. Elle modifie l'administration et la gestion quotidienne de l'enseignement de manière prégnante. De par ses caractéristiques intrinsèques, elle suppose également un apprentissage à travers le temps et l'espace. Devant ces multiples enjeux, nous avons analysé les usages de la tablette en salle de classe auprès de 200 enseignants de 45 établissements scolaires au Québec. Les résultats mettent en évidence que l'utilisation de la tablette induit des usages pédagogiques novateurs qui facilitent l'enseignement et favorisent l'apprentissage des élèves. Cependant, nous constatons que la tablette n'est pas utilisée à son plein potentiel et que certains usages devraient être envisagés selon une perspective plus efficiente et adaptée. Le présent article, issu de «L'intégration des TIC en contexte éducatif: modèles, réalités et enjeux » (Fiévez, 2017), synthétise l'ensemble des réalités pédagogiques étudiées et apporte des éléments de réponse pour les décideurs et les différents acteurs de l'éducation.

Mots-clés

Apprentissage mobile, tablette, enseignant, usages, activités, modèle, ubiquité.

Abstract

The use of tablets in the school context is becoming more visible in classrooms in North America and Europe. The integration of this new technology induces changes in teaching and learning activities. The tablet brings many new educational opportunities and educational uses. It fundamentally changes the administration and daily management of education. Due to its intrinsic characteristics, it also involves learning through time and space. Given these multiple realities, we have analyzed the uses of the tablet in classrooms with 200 teachers from 45 schools. The results show that the use of the tablet induces innovative teaching practices that facilitate teaching and promote student learning. However, we find that the appropriation of the tablet is an ongoing process and that new implementations, as for those existing, must feed into a careful analysis of the ramifications of practices mediated by the integration of this tool.

\section{Keywords}

Mobile learning, tablet, teacher, customs, activities, model, ubiquity.

\section{Introduction}

Aujourd'hui, dans le paysage francophone, 480000 élèves utilisent quotidiennement une tablette en salle de classe. En quelques années, celle-ci est devenue de plus en plus présente dans les ménages de l'OCDE (OCDE, 2015). De manière encore plus visible, nous constatons une prédominance marquée de la tablette dans les salles de classe au primaire, au secondaire, au collégial et à l'université (International Data Corporation, 2014). Il est donc légitime que cet outil soit un point central de recherche et de discussions dans les milieux scientifiques et de pratique (Wishard,2015). L'utilisation d'un artéfact technologique comme la tablette dans une salle de classe présuppose la prise en considération et la modification des genèses instrumentales et ergonomiques, une modification des pratiques enseignantes et des conceptualisations de ces pratiques (Pellerin,2015). Certains parleront même d'un nouveau paradigme éducationnel basé sur des perspectives nouvelles, complémentaires, voire augmentées, en comparaison à un enseignement traditionnel (Penny, Shugar, McConatha, Bolton et Taylor, 2013). Après plusieurs années d'utilisation en contexte éducatif, considérant les nombreux avantages explicités par divers auteurs (Henderson et Yeow, 2012; Villemonteix et al., 2014) et les inconvénients mis en évidence (Hutchison, Beschorner et SchmidtCrawford, 2012), pour quelles activités cet outil est-il utilisé? Quels sont les aboutissants de cette utilisation? Le présent article explore quantitativement et qualitativement les usages pédagogiques des tablettes en contexte éducatif. Il apporte des éléments de réponse concernant une technologie trop souvent considérée comme un outil unique et transformateur pour l'enseignement, et non comme un outil complémentaire doté d'un potentiel cognitif spécifique. À notre avis, après une analyse exhaustive, il s'agit d'un outil qui doit rester - avant toute chose - au service de l'éducation et qui doit répondre aux besoins éducatifs de l'école. Le but de cet article est d'identifier les aboutissants 
de l'utilisation de la tablette en salle de classe afin, comme cela a été fait dans Fiévez (2017), d'analyser les tenants de son intégration.

\section{Question de recherche}

La question de recherche est la suivante : quelles sont les pratiques pédagogiques liées à l'utilisation de la tablette en contexte scolaire? La réponse à cette question implique une analyse des activités, des applications et des usages de la tablette en salle de classe par les enseignants. Cette étude est donc à prédominance qualitative et exploratoire. Pour cela, et afin de circonscrire le contexte et les implications de ces usages, un cadre théorique spécifique a été élaboré. Il permet de circonscrire notre objet de recherche et de l'analyser d'un point de vue théorique, avant de le confronter aux réalités de terrain.

\section{Cadre théorique}

\section{Le concept d'usage appliqué aux technologies}

Le concept d'usage est très souvent utilisé en sciences de l'éducation pour repérer, décrire et analyser des comportements (Boudokhane, 2006). Pour Proulx et Breton (2002), le terme « usage » renvoie à de multiples définitions : celles-ci se situent souvent entre l'adoption (achat, consommation et utilisation d'un outil technologique), l'utilisation (maîtrise technique et cognitive de l'outil) et l'appropriation (conception d'innovations pédagogiques). Par l'usage d'un outil technologique, on vise principalement les pratiques d'intégration et d'identification des usages des TIC. Elles sont analysées en fonction de leur fréquence d'utilisation, de l'intensité d'utilisation et de leur intégration dans les pratiques personnelles, professionnelles et pédagogiques de l'enseignant (Proulx, 2004).

Différents types d'usages généraux peuvent être exposés: des usages personnels, des usages professionnels et des usages pédagogiques, et ceux-ci ne se construisent pas forcément selon un ordre linéaire (Raby, 2004). Aussi, nous pouvons discerner plusieurs types d'usages spécifiquement liés aux contextes d'intégration (Bernet et Karsenti, 2013; Tondeur et al., 2012) : usages comme éléments de compétences techniques, outil d'information, outil d'apprentissage ou encore outil ludique.

\section{Les usages de la technologie mobile}

De par notre définition, basée sur l'analyse et la combinaison des travaux de David et al. (2007), de Fernández-López, Rodríguez-Fórtiz, Rodríguez-Almendros et Martínez-Segura (2013), de Kukulska-Hulme et Traxler (2005) et d'O'Malley et al. (2005), la tablette est « un appareil mobile, personnalisable et de taille souvent réduite pourvue d'une interface, dont les fonctionnalités se situent à l'intersection de l'ordinateur portable et du téléphone intelligent ». Cette définition technique nous permet de mettre l'accent sur l'aspect tactile et de spécifier que cet outil est différent d'un ordinateur.

Ensuite, au-delà des caractéristiques techniques, cet outil nécessite un questionnement sur les tenants et aboutissants de son intégration pédagogique. Il faut impérativement mettre en évidence que les deux 
entités (technique et pédagogique) sont intrinsèquement liées et qu'elles interagissent entre elles lors de l'intégration de l'outil (Benson, 2013). Ainsi, considérant les caractéristiques mobiles et portables de l'outil, deux concepts centraux de son utilisation en salle de classe se dévoilent : l'apprentissage mobile et l'apprentissage ubiquitaire. Ces concepts doivent nécessairement être explicités, car ils représentent des éléments essentiels de l'utilisation de la tablette et de son potentiel.

L'apprentissage mobile est basé sur une technologie qui encadre l'apprentissage à travers le temps et l'espace, où l'apprentissage prend les avantages, les opportunités et les défis de l'appareil mobile utilisé, incluant la mobilité, la complémentarité et l'efficience liée à son utilisation (David et al., 2007; Kukulska-Hulme et Traxler, 2005). Dans le prolongement de l'apprentissage mobile, un autre type d'apprentissage plus complexe existe. De fait, l'apprentissage ubiquitaire exploiterait à son plein potentiel les outils mobiles. Celui-ci met en ouvre des processus qui ont lieu au sein d'une activité mobile (et d'un contexte plus large que celui de la classe), mais qui, à cette fin, met en œuvre différents outils (Gicquel, 2010). En corolaire, de par ses potentialités et caractéristiques, l'apprentissage mobile s'intègre dans trois dimensions afin d'obtenir un usage réfléchi et efficient. La première dimension vise la personnalisation, où l'apprenant s'approprie la technologie selon les principes de la théorie socioculturelle de Vygotski (voir Bruner, 1985) et de la motivation (Pintrich et Schunk, 2002). L'apprenant s'approprie l'outil, personnalise son apprentissage en autonomie, favorisant ainsi différentes méthodes et approches. La deuxième dimension, l'authenticité, situe l'apprentissage dans une des tâches signifiantes, contextualisées et concrètes qui ont un impact direct sur l'environnement de l'élève. La troisième dimension vise la collaboration à travers le temps et l'espace où l'apprenant utilise l'outil dans un environnement collaboratif avec une communication multimodale soutenue entre les pairs. En termes d'usages, l'apprentissage mobile demande des tâches et des processus authentiques dans des contextes riches et diversifiés en fonction des besoins des utilisateurs (Laroussi, 2012). Ces usages sont soutenus par des ressources telles que la géolocalisation, l'accès à Internet, les ressources, la réalité augmentée, la formation en ligne et la formation présentielle (Nincarean, Alia, Halim et Rahman, 2013). Cette combinaison entre la formation en ligne et la formation en présentiel est illustrée par le concept de blended learning, ou apprentissage hybride (voir la figure 1) qui se divise en quatre modèles. Le premier, le modèle dit de "rotation ", se fonde sur une variation des modalités d'apprentissage, dont une au moins se fait en ligne. Ce modèle inclut par exemple le modèle de la classe inversée, très détaillé dans la littérature, où les projets se réalisent en classe et où les savoirs magistraux se déroulent en ligne. Le deuxième modèle est celui du modèle "flexible ", où le contenu et les instructions se font prioritairement en ligne, mais la formation en présentiel est flexible en fonction des besoins de l'apprenant. Ensuite, le modèle « multimodal personnel » se base sur un scénario dans lequel les élèves choisissent de suivre un ou plusieurs cours entièrement en ligne pour compléter leurs cours traditionnels. Enfin, le modèle «virtuel enrichi » vise l'expérience globale de l'école dans laquelle les élèves partagent leur temps entre la fréquentation d'un établissement et l'apprentissage à distance. Les deux premiers modèles sont utilisables dans l'enseignement secondaire, alors que les deux derniers visent plutôt l'enseignement supérieur.

Ces différentes modélisations et théories sont explicitées dans la figure 1 afin d'illustrer les potentialités de loutil et de comprendre si celles-ci sont visibles empiriquement dans les salles de classe. Il s'agit de vérifier si l'outil en tant que tel, dans sa perspective mobile et ubiquitaire, met en évidence des méthodes nouvelles et complémentaires. De cette manière, nous pouvons situer avec plus de précision la place de la tablette dans la salle de classe. 


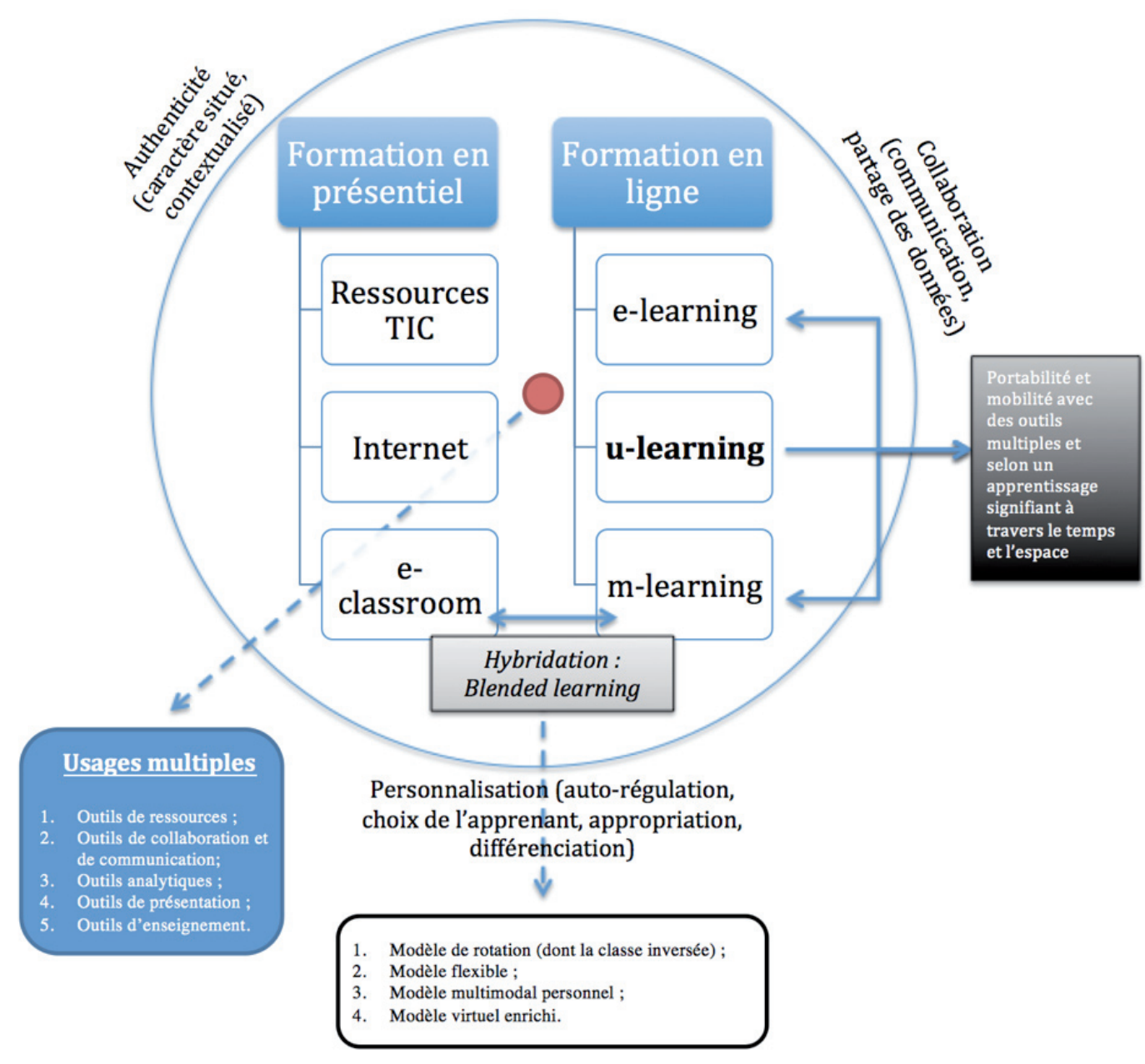

\section{Figure 1}

La place de l'apprentissage mobile dans le contexte éducatif (Fiévez, 2017, adapté de Derycke, 2006; Kearney, Schuck, Burden et Aubusson, 2012; Liaw, Hatala et Huang, 2010; Park, 2011; Staker et Horn, 2012).

Considérant que l'apprentissage informel représente plus de $80 \%$ de tous les apprentissages (Sockett et Kusyk, 2012), l'outil mobile apporte de nouvelles perspectives pour la formation en ligne et pour l'ubiquité, créant ainsi de nouvelles perspectives d'enseignement et d'apprentissage. Comme l'indique Park (2011), l'apprentissage mobile ajoute une nouvelle dimension collaborative, des interactions sociales et un échange rapide des données. La caractéristique la plus prégnante est un apprentissage à travers le temps et l'espace. 


\section{Les usages pédagogiques de la tablette en salle de classe}

La fréquence d'utilisation de la tablette en salle de classe dépend en grande partie de la compréhension des enseignants de cette technologie, du potentiel de l'outil et des usages réalisés. Pour Walters et Baum (2011), la tablette n'est pas seulement un outil de consommation, elle est également bénéfique pour la création d'idées et de contenu. Quant à Benson (2013), il ajoute que l'utilisation des technologies mobiles par les jeunes générations devrait contribuer à former des apprenants créatifs et communicants, plutôt que des consommateurs passifs. Ce dernier propose donc de reconfigurer les salles de classe en espaces plus ouverts, combinant présence physique et collaboration distante. De manière générale, comme l'expliquent Churchill et Wang (2014), nous constatons que l'utilisation de la tablette passe souvent par une utilisation singulière et peu combinée (voire complémentaire) à d'autres technologies (projecteur, ordinateur ou tableau blanc interactif). Pour cela, l'enseignant devra rechercher et trouver les applications et usages à privilégier dans sa salle de classe (Vu, 2013). Il devra aussi combiner les caractéristiques de l'outil, les applications, les ressources disponibles, les avantages et les limitations de la tablette.

Pour ce faire, la littérature recensée met en corrélation les caractéristiques de la tablette et les usages observés. Ainsi, différentes caractéristiques présenteraient une différence significative sur les usages proposés en salle de classe avec un autre outil : l'écran, la batterie de longue durée, la rapidité de l'outil, la portabilité et l'accès instantané aux ressources (Bansavich, 2011; Beebe, 2011). En corolaire aux caractéristiques techniques, de multiples usages sont recensés : réaliser des tâches collaboratives (Beauchamp et al., 2014; Lederman et Abell, 2014); élaborer des tutoriels et des capsules vidéo (Giroux, Coulombe, Cody et Gaudreault, 2013); utiliser les technologies mobiles dans un environnement authentique et réaliser des activités à l'extérieur de la salle de classe (Burden et Maher, 2014; Fabian et MacLean, 2014; Henderson et Yeow, 2012; Kinash, Brand et Mathew, 2012; Villemonteix et al., 2014); utiliser la tablette pour lire et écrire (Hutchison et al., 2012; Northrop et Killeen, 2013; Rhodes, 2013); permettre les activités interactives et de production (Ostler et Topp, 2013); accéder à de l'information, éditer et partager de l'information (Babnik et al., 2011; Hutchison et Beschorner, 2014). Enfin, il est également nécessaire de considérer que cet outil est souvent utilisé dans un contexte extérieur (à la maison ou sur la route) et que cet artéfact entre, de facto, comme illustré dans la figure 1, dans une dimension mobile et ubiquitaire (Peluso, 2012). In fine, les activités doivent être variées, pertinentes et en relation avec les objectifs d'apprentissage (Fabian et MacLean, 2014; Montrieux, Vanderlinde, Courtois, Schellens et De Marez, 2014). Lorsque nous abordons le concept d'usage, les applications technologiques utilisées lors de ces usages arrivent indubitablement dans l'équation.

\section{Les applications liées aux usages de la tablette}

Les tablettes donnent accès à un nombre important d'applications dans différents domaines d'expertise. Cependant, l'enseignant doit déterminer quelles sont les applications les plus pertinentes et celles qu'il peut utiliser dans ses cours, car certaines correspondent ou non à un usage pédagogique (Shah, 2011). Comme le mettent de l'avant Bruce, Bourbous, El-Chami, Eliot et Howard (2012), l'enseignant, même selon ses propres latitudes pédagogiques, est lié par la disponibilité des applications et leurs fonctionnalités. À l'instar de Boéchat-Heer (2014) et de Churchill et Wang (2014), nous pouvons 
mettre en évidence différents axes pédagogiques développés par l'utilisation de la tablette (l'iPad, dans le cas de notre étude) en contexte scolaire : les applications de consultation, de collaboration, de production, de partage de contenu et d'enseignement. Il serait hasardeux et complexe de calculer la proportion d'applications utilisées par les enseignants selon leurs axes de classification. De manière globale, les applications induisent des usages différents en fonction de leurs particularités, mais aussi en fonction des activités réalisées par l'enseignant et de ses choix didactiques. Les applications les plus utilisées par les enseignants sont Keynote, Antidote, SimpleMind, Explain Everything, Edmodo, PDF Notes, Splashtop, Dropbox, les baladodiffusions et les codes QR (Babnik et al.,2011; Gesser, 2011; Giroux et al., 2013; Karsenti et Fiévez, 2013; Murray et Olcese, 2011). Ainsi, ces trois dernières années, les auteurs signalent que l'usage principal de la tablette est l'accès à l'information et le partage de celle-ci.

\section{Méthodologie}

Le cadre théorique présenté plus haut met en évidence les réalités pédagogiques et techniques entourant les usages de la tablette en contexte éducatif. En continuité, nous pouvons établir une méthodologie précise afin de répondre à l'objectif énoncé plus haut.

\section{Participants}

Les participants de cette étude sont des enseignants des écoles privées et publiques du Québec. L'échantillonnage est basé sur la population accessible, c'est-à-dire la portion de la population que l'on peut atteindre. Nous avons contacté 18 écoles, publiques et privées, qui utilisent de façon quotidienne une tablette et selon un environnement 1:1 (une tablette par élève). Nous avons privilégié un échantillonnage aléatoire stratifié proportionnel en deux strates. La première strate de 100 participants a été prélevée aléatoirement dans les différentes écoles privées participantes. La seconde strate de 100 participants a été prélevée aléatoirement dans les différentes écoles publiques participantes. Le but était d'obtenir un échantillon plus représentatif que l'échantillonnage aléatoire afin de limiter les erreurs (Portney et Watkins, 2009). Grâce à d'autres recherches déjà menées, nous avons une excellente connaissance de la population cible, ce qui nous a permis de choisir le plus adéquatement possible les variables de stratification. Au total, 200 enseignants (40,96 \% d'hommes, 59,04 \% de femmes) de toutes les disciplines scolaires ont participé à l'étude (figure 3). Comme le montre la figure 2, cette recherche a été réalisée dans les écoles secondaires où les enseignants travaillent principalement en première, deuxième et troisième année du secondaire (71\%). 


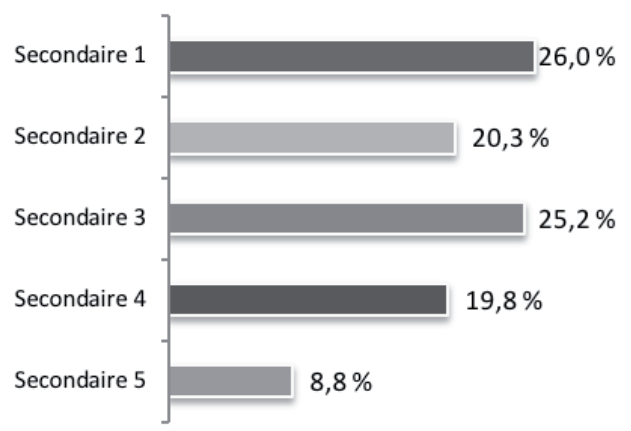

Figure 2

Répartition des enseignants selon leur niveau d'enseignement.

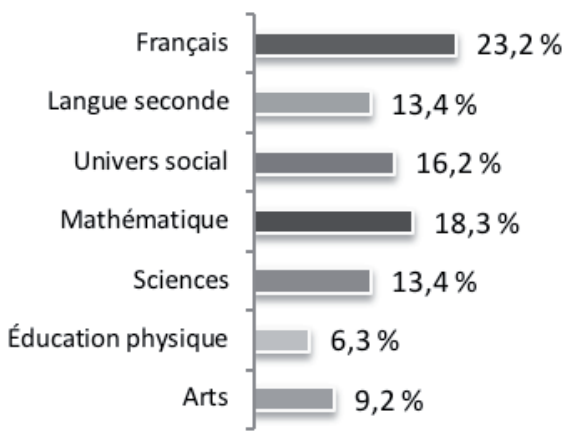

Figure 3

Matière enseignée par les enseignants ayant participé à l'étude.

\section{Collecte de données}

Les données recueillies proviennent des questionnaires administrés aux enseignants $(n=200)$ par le chercheur via une plateforme de sondage (SurveyMonkey). Il s'agit de données quantitatives et qualitatives suivant la méthodologie mixte. La recherche entreprise compte trois principaux instruments de collecte de données: questionnaires d'enquête en ligne auprès des enseignants $(n=200)$; entrevues semi-dirigées auprès des enseignants $(n=10)$; entrevues individuelles semidirigées auprès des enseignants $(n=10)$. Les questionnaires ont été validés et ajustés en fonction des recherches précédentes de Karsenti et Collin (2011) et de Raby, Karsenti, Meunier et Villeneuve (2011), où les auteurs mettent en évidence les activités didactiques possiblement réalisées à l'aide d'un outil technologique. Le protocole des entrevues individuelles semi-dirigées et des entrevues de groupe semi-dirigées reprenait les catégories de questions des questionnaires et visait à approfondir et à mettre en relation les principales tendances relevées. Les entrevues individuelles ont notamment permis de recueillir les perceptions des enseignants à l'égard de nos objectifs de recherche et de préciser les résultats obtenus. Les entrevues ont aussi permis d'approfondir notre compréhension de certains usages de la tablette en salle de classe.

\section{Analyse des principaux résultats}

Les données issues des questionnaires sont constituées à la fois de données qualitatives et de données quantitatives. L'analyse des données qualitatives a été effectuée par codage, suivant les principes de l'analyse de contenu (L'Écuyer, 1990; Van der Maren, 1996). Les analyses ont été réalisées à l'aide du logiciel QDA Miner, très utilisé dans l'analyse de données qualitatives en recherche (voir Derobertmasure et Robertson, 2013; Fielding, 2012; Karsenti et al., 2011). Nous avons réalisé l'analyse qualitative en nous basant sur les étapes méthodologiques de Royer, Baribeau et Duchesne (2009) et de Dépelteau (2011), mais aussi sur les travaux de Bogdan et Biklen (2003), concernant l'élaboration de la grille de codes. Concernant les analyses quantitatives, les logiciels Excel 14.5 et SPSS 22.0 ont 
été utilisés afin de réaliser des statistiques descriptives. En fin d'analyse, afin d'assurer la validité, un contre-codage a été appliqué à $25 \%$ des unités de sens. Le taux d'accord moyen est de $82 \%$ entre les codeurs.

\section{Résultats}

\section{Expérience préalable des enseignants}

Afin de situer les usages des technologies avant l'intégration de la tablette, nous avons demandé aux enseignants quelles étaient leurs expériences préalables quant à l'utilisation d'un outil technologique. Les résultats montrent que 77,2\% des enseignants n'avaient «jamais ou très rarement " utilisé la tablette avant que l'expérience ne soit lancée dans leur école. Seulement 11,1\% des enseignants l'avaient utilisée à quelques reprises et 11,6 \% l'avaient utilisée de façon plus régulière. Nous avons également demandé aux enseignants depuis combien de temps ils utilisaient les technologies en salle de classe. Nous constatons que la moitié d'entre eux ont une utilisation récente des outils technologiques. Ainsi, $54,4 \%$ des enseignants ont d'une seule à cinq années d'expérience (soit la durée du projet « tablettes " actuel). À l'opposé, seulement 16,0\% des enseignants ont de 6 à 9 années d'expérience avec les outils technologiques et 20,7\% ont plus de 10 ans d'expérience. Ces résultats montrent que les enseignants ont une préparation plutôt sommaire de l'utilisation d'une tablette. Les causes sont multiples : nous pouvons mettre en évidence le caractère récent de l'outil (l'iPad a été créé en 2010) et une arrivée tardive auprès des enseignants utilisateurs. En moyenne, les enseignants ont reçu les tablettes 6 mois avant l'arrivée de l'outil auprès des élèves avec et parfois sans formation (technique ou pédagogique) préalable. D'autre part, lors des entrevues, les enseignants ont également nuancé leurs constats concernant leur appropriation technopédagogique. Premièrement, ils expliquent que, lors de leur formation commune en début et en cours de projet, les rudiments techniques étaient privilégiés par rapport à l'appropriation pédagogique de l'outil. Les enseignants expliquent également que l'introduction de la tablette dans la salle de classe a significativement augmenté la collaboration entre l'enseignant et les élèves. Ils expliquent que les enseignants et les élèves participent de concert à l'intégration de l'outil et à son appropriation dans la salle de classe. In fine, l'utilisation préalable de l'outil s'est révélée sommaire, tant d'un point de vue technique que pédagogique, pour les enseignants, même si des perspectives collaboratives ont été constatées.

\section{Usage des outils technologiques en salle de classe}

Notre recherche montre également que $96,7 \%$ des enseignants utilisent d'autres outils technologiques en complément de la tablette : $25,9 \%$ des répondants utilisent un ordinateur fixe en salle de classe ou un ordinateur portable $(31,9 \%)$ en plus de la tablette; 7,0 \% des enseignants utilisent un téléphone portable et 13,5\% d'entre eux utilisent un tableau blanc interactif (TBI).

"Personnellement, je vais faire mes ebooks sur l'ordinateur, car c'est plus facile et je les envoie aux élèrves ou je les utilise avec le TBI. » $(\mathrm{EN} 3,2015)$

"J'utilise beaucoup mon portable [téléphone intelligent] en classe, pour contrôler les Keynote, pour communiquer ou chercher des ressources. »(EN4, 2015) 
Les entrevues viennent compléter les données quantitatives : elles révèlent que les enseignants utilisent d'autres outils technologiques comme l'ordinateur fixe, l'ordinateur portable, le TBI et le téléphone intelligent. Nous observons que le choix de l'outil technologique se fait en fonction de ses potentialités et de ses implications. Par exemple, l'ordinateur vient compléter la conception des séquences de cours et participe à la réalisation de tâches plus complexes (outils mathématiques ou graphiques). Le TBI, quant à lui, vient ajouter un soutien à l'enseignement et un outil collaboratif pour les élèves.

\section{Temps d'utilisation de la tablette en salle de classe}

Notre étude démontre aussi que la tablette est assez utilisée en salle de classe. En effet, les enseignants indiquent que les élèves utilisent la tablette au minimum 30 minutes $(21,5 \%)$ par cours et que 37,5\% des enseignants l'utilisent en classe de 15 à 29 minutes. Au total, $59 \%$ des enseignants utilisent la tablette plus de $50 \%$ du temps de cours. Ces résultats sont largement appuyés par les entrevues réalisées auprès des enseignants, et ce, même si des nuances sont apportées. En effet, des différences de fréquence apparaissent en fonction du professeur, de son implication dans le projet et de la matière enseignée. Selon les considérations pédagogiques de l'enseignant, certains utilisent la tablette lors de chaque séance de cours et pendant toute la période, alors que d'autres enseignants l'utilisent plus sporadiquement :

«[...] Moi, personnellement, j'essaie de trouver des façons de l'exploiter le plus possible, pour les projets par exemple, là, je cogite mes projets pour que la tablette soit au centre de l'apprentissage. » (EN2, 2015)

«[...] vouloir utiliser la tablette à tout prix, ce n’est pas une bonne idée. Il y a certains travaux que ça aide, comme en sciences [...], et d'autres pas.» (EN8, 2015)

\section{Usages de la tablette en salle de classe}

Les analyses quantitatives et qualitatives révèlent plusieurs aspects concernant les usages de la tablette en salle de classe par les enseignants. Les résultats aux questionnaires explicitent les usages de ces applications en salle de classe (voir la figure 4). Lorsque nous analysons les résultats, nous pouvons mettre en relation les usages et les applications liés. Ainsi, certaines applications induisent des usages spécifiques, mais il est nécessaire d'examiner les usages déclarés afin d’obtenir un portrait exhaustif de l'utilisation de la tablette en salle de classe. 


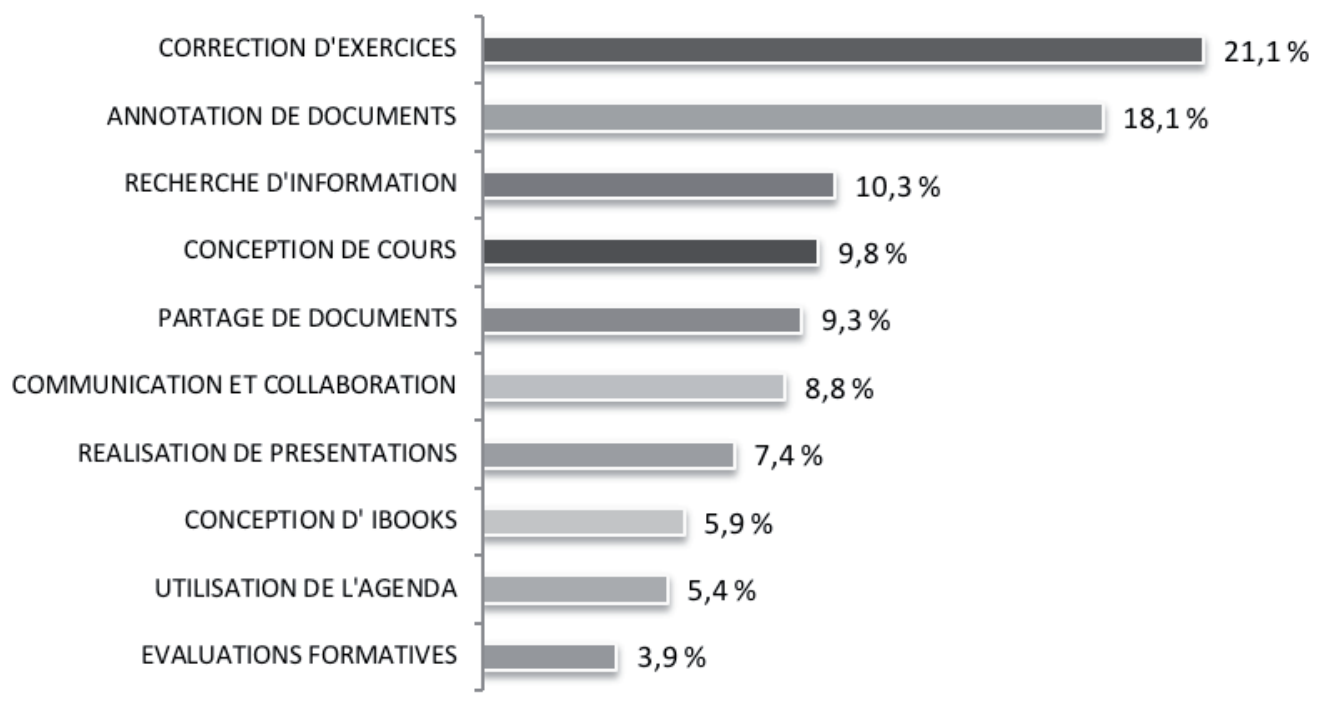

\section{Figure 4}

Principaux usages pédagogiques de la tablette par les enseignants.

\section{Les activités d'écriture et d'annotation}

À propos des usages, les enseignants spécifient qu'ils utilisent la tablette pour la correction des exercices $(21,1 \%)$ et plus précisément pour la vérification des travaux et des devoirs. D'autres usages visent la rédaction, l'annotation et la conception de documents. Pour le travail entourant la rédaction, les enseignants indiquent qu'ils utilisent la tablette pour rechercher l'information nécessaire au texte produit, pour faire collaborer les élèves entre eux et pour diffuser le texte. Dans une moindre mesure, la tablette est utilisée pour la rédaction pure.

"Lors de la rédaction d'une dissertation, je demande aux élèves d'utiliser la tablette pour faire la rédaction au propre et pour me l'envoyer [...] ils peuvent utiliser le papier s'ils veulent et en moyenne c'est ce quills font pour le brouillon.» (EN26, 2015)

L'analyse des entrevues montre que les tablettes complexifient certaines tâches d'écriture. En effet, les enseignants indiquent que le fait que les applications soient séparées demande à l'utilisateur de « jongler » avec l'ensemble des icônes. Ils doivent souvent copier-coller le texte ou le vérifier d'une application à l'autre, ce qui complexifie les rédactions et la correction du document. À cette fin, l'application la plus utilisée pour la correction orthographique est l'application Antidote, bien que différente du logiciel usuel sur ordinateur. Enfin, pour certaines tâches d'écriture, les enseignants indiquent que l'utilisation du papier est parfois nécessaire dans certains cours. 


\section{Le transfert des documents}

Le transfert des documents est réalisé entre l'enseignant et les élèves lors de la remise de travaux et de leur correction ou encore lors de la diffusion des supports de cours. Un partage des documents est également réalisé entre les enseignants afin de partager les différentes ressources pédagogiques. Ces échanges peuvent se faire au sein de l'équipe éducative (enseignants, conseillers, direction), mais aussi avec des acteurs extérieurs (RÉCIT, conseillers pédagogiques, enseignants ou autres). Une application très utilisée par les enseignants pour le partage des documents est l'application Google Drive et Dropbox.

"Je pense qu'au niveau de l'organisation de la matière, ça va aider aussi, dans le sens quion organise le cours, sur ChallengeU ou sur Dropbox, j'utilise les deux plateformes de la même façon [...]. » (EN12, 2015)

Nous constatons également que les enseignants utilisent certaines plateformes de partage, de création de contenu et de consultation comme ChallengeU, très utilisée dans les écoles participantes. Les enseignants vont y déposer les supports de cours sur une page prévue à cet effet et les élèves y accèdent en salle de classe et à domicile, favorisant ainsi l'apprentissage ubiquitaire. Les enseignants ajoutent également que ce type de plateformes favorise l'organisation du travail de l'enseignant et facilite la consultation des supports de cours pour les élèves.

\section{La communication et la collaboration avec la tablette}

La communication entre les enseignants et entre les élèves et l'enseignant a considérablement augmenté avec la tablette. Les enseignants communiquent et collaborent entre eux afin de partager les ressources et supports de cours. Ils communiquent également sur différents points d'enseignement et d'organisation (réunion, planification, etc.). Nous constatons aussi une communication et un échange importants entre les élèves et l'enseignant. Ce dernier a redéfini son rôle de médiateur à l'extérieur de la classe, où il est beaucoup plus présent. Nous constatons une forte présence de l'ubiquité dans la communication entre l'enseignant et sa classe.

"ils font leurs devoirs dans le livre, en version électronique, screenshot, puis ils envoient ça dans Notability, ils l'envoient ensuite par e-mail. Entre collègues, c'est plus facile de communiquer, on a iMessage et FaceTime pour se rejoindre même si on est dans des locaux différents, c'est plus facile de parler avec mes collègues. » (EN11, 2015)

En continuité, les enseignants ont constaté que les élèves ne maîtrisent pas toujours les usages pédagogiques pertinents qu'ils devraient privilégier avec la tablette. Les jeux ou les réseaux sociaux sont souvent utilisés en salle de classe et pour des activités non éducatives. Ce point représente un défi majeur pour les enseignants et la gestion de classe est parfois complexifiée.

Conception de cours et manuels scolaires

La conception de séquences de cours (10,2\%) est la quatrième activité réalisée par les enseignants. Ceux-ci conçoivent les cours sur différents supports comme les PDF ou les livres numériques (eBook). Ils utilisent également les manuels scolaires (7,0 \%) afin de soutenir l'enseignement et l'apprentissage. Ainsi, les enseignants indiquent que l'utilisation de manuels scolaires dans les différents niveaux d'enseignement est récurrente. 
"Tout le monde a le livre numérique, donc là,je peux projeter mon livre à moi et annoter en même temps. [...] là, pour eux, c'est concret, ils le voient. Ils sont d'ailleurs beaucoup plus autonomes. » (EN21, 2016)

Cependant, une proportion réduite des manuels scolaires numériques sont utilisés, car certains ne sont pas encore suffisamment aboutis pour une utilisation aisée et efficiente en salle de classe. De ce fait, certains manuels scolaires sont utilisés en format papier. En complément aux manuels utilisés, les cahiers d'exercices sont remplis en grande partie en format papier, pour les mêmes raisons techniques.

\section{La recherche et l'accès à l'information}

Un des principaux usages de la tablette en salle de classe est la recherche de l'information. L'accès immédiat et permanent aux informations présentes sur Internet permet aux enseignants d'utiliser les ressources en tout temps, soit pour leur propre accès, soit pour celui des élèves. L'analyse de leurs réponses montre qu'ils apprécient grandement le fait d'avoir le contrôle sur cet accès, sur l'organisation et la modification de l'information et sur sa diffusion :

"On fait beaucoup de recherches sur différents sujets, que ce soit un pays, une ressource naturelle ou autre chose. Aussi, l'apprentissage par des capsules de tutorat, l'élève, il y a accès tout le temps, donc s'il a des questions ou s'il n'a pas bien compris, il peut aller chercher l'information en revoyant la capsule.» (EN87, 2015)

\section{Les évaluations formatives}

En début de cours, les enseignants expliquent qu'ils réalisent des évaluations formatives (3,9\%) afin de cibler le niveau et la compréhension (individuelle ou collective) des élèves dans la matière enseignée. Pour ce faire, ils utilisent l'application Socrative ou Kahoot! :

"En début de cours, je fais un quiz combiné à des interactions. Par exemple, avec notre tableau interactif en avant, j'utilise Socrative, une application qui permet de faire des quiz, de voir des résultats en temps réel et qui a une bonne réception du côté des étudiants. » (EN28, 2015)

\section{La portabilité et l'apprentissage ubiquitaire}

Il est important d'ajouter l'élément intégrateur de la tablette: l'apprentissage mobile. Comme le montrent les résultats, les enseignants n'évoquent que très peu (5\%) une utilisation mobile de la tablette dans son aspect portable. Ainsi, les usages à l'extérieur de la salle de classe reviennent rarement dans les questionnaires et les entrevues. De fait, seulement deux enseignants ont évoqué la question :

"Je demande aux élèves de faire des rallyes à l'extérieur de la classe, par exemple, en éthique, pour les signes religieux (églises, etc.) les élèves vont dans le quartier et réalisent un portfolio qu’ils présentent à la classe. Ils peuvent utiliser l'iPad ou leur portable, si la qualité photo est bonne. " (EN68, 2015)

\section{Discussion}

En corrélation avec les études précédentes (Karsenti et Collin, 2011; Karsenti et Fiévez, 2013; PaladinoChristin, 2015), les résultats montrent que les enseignants utilisaient peu les outils technologiques avant l'introduction de la tablette dans leur établissement scolaire. Aussi, la formation était peu présente avant 
le début de l'intégration de la tablette. Et si cette formation était présente, elle possédait une visée plus technique que pédagogique. Pourtant, comme l'explique Benson (2013), il est nécessaire de combiner ces deux entités afin d'obtenir une intégration efficiente.

De manière générale, à l'instar de Churchill et Wang (2014), les enseignants se basent sur des usages existants où les technologies sont déjà utilisées de manière efficiente en contexte scolaire. Ensuite, ils puisent les différents usages dans les formations reçues, dans les communautés de pratique ou chez les pairs, et ils adaptent ces usages à leur propre pratique (Beauchamp et al., 2014; Flewitt, Kucirkova et Messer, 2014; Underwood et Dillon, 2011; Villemonteix et al., 2014). La fréquence d'utilisation de la tablette est tributaire de ces pratiques. Ainsi, le temps d'utilisation varie en fonction des activités et des enseignants. Certains enseignants utilisent la tablette au cours d'une séance complète, comme un outil intégrateur (utilisée pour toutes les activités d'apprentissage). D'autres enseignants l'utilisent uniquement pour une activité ciblée sur une courte période de temps. À ces usages spécifiques viennent s'ajouter les plateformes de partage et de diffusion de documents qui soutiennent l'enseignement et la collaboration entre les élèves, mais aussi la communication avec l'enseignant (Lederman et Abell, 2014). En continuité, et en adéquation avec les travaux de Huber (2012), les résultats montrent que l'appropriation de la tablette en salle de classe passe également par une collaboration avec les élèves quant à l'appropriation technique de la tablette et aux nouvelles applications présentes sur le Store. En effet, les élèves conseillent les enseignants et apportent un soutien collaboratif aux cours enseignés, comme illustré dans les travaux d'Henderson et Yeow (2012).

Ensuite, sur le plan des usages spécifiques de la tablette en contexte scolaire, comme le montre la figure 4, l'annotation des documents et la correction des travaux des élèves sont les activités principales réalisées par les enseignants. Ces résultats sont peu présents dans la littérature, l'usage principal relevé étant la recherche de l'information. Il s'agit ici d'un point intéressant, car nous constatons une modification structurelle de l'utilisation de la tablette par l'enseignant, passant d'un outil de consultation à un outil de production. Comme nous l'avons montré et comme la littérature le met largement en évidence (Babnik et al., 2011; Gesser, 2011; Murray et Olcese, 2011; O'Sullivan, 2012), l'usage principal de la tablette est l'accès à l'information. L'enseignant accède en tout lieu et en tout temps à l'information, modifiant ainsi son rôle de diffuseur de savoir pour entrer dans une approche plus collaborative de l'enseignement et de l'apprentissage. Cet aspect se situe dans notre étude en seconde position. Ensuite, la conception de cours, combinée à la conception d'eBook, est une activité centrale du rôle de l'enseignant (Kearney et Maher, 2013); elle arrive cependant en $4^{\mathrm{e}}$ position des usages de la tablette. Ici encore, la production de contenu est visible, mais en proportion réduite par rapport aux activités de consultation. Le partage des documents, également explicités par certains auteurs (Alyahya et Gall, 2012; Gesser, 2011; Mockus et al., 2011), est visible dans notre étude. De fait, les enseignants partagent leurs supports de cours avec les élèves et ces derniers complètent leurs documents qu'ils déposent ensuite sur les plateformes de partage (Google Docs ou Dropbox). La collaboration entre les apprenants signalée par Henderson et Yeow (2012) est également visible chez les enseignants où ces derniers échangent leurs supports de cours et communiquent avec leurs collègues. La place de l'évaluation formative avancée par Benson (2013) se trouve ici vérifiée lors des quiz avec Socrative. De son côté, la présence de la prise de notes et de l'utilisation d'exerciseurs en ligne est peu décrite dans la littérature, mais bien présente dans notre étude.

Il est également important d'analyser la tablette dans sa perspective mobile, voire ubiquitaire et de comprendre que malgré les nombreux avantages mis en évidence par la littérature (Bansavich, 2011; 
Beebe, 2011), peu d'enseignants utilisent la tablette en dehors de la salle de classe pour des activités extérieures ou supplémentaires. L'utilisation de la tablette en salle de classe demande encore de nombreux ajustements pédagogiques afin de l'exploiter à son plein potentiel.

\section{Conclusion}

En conclusion, quels sont les usages de la tablette en salle de classe et comment se positionne-t-elle dans le paradigme de l'apprentissage mobile? Si nous reprenons le schéma de la figure 1 et que nous l'adaptons à la lumière de nos résultats, nous obtenons une conclusion synthétique et modélisée de l'utilisation de la tablette par les enseignants (figure 5). Ainsi, comme de nombreux auteurs, tels Derycke (2006), Michel et al. (2012), Milot (2010) et O'Sullivan (2012), l'ont mis en évidence, la tablette permet d'introduire le paradigme de l'apprentissage mobile, mais ne l'exploite pas à son plein potentiel. Elle permet de favoriser le nomadisme chez l'apprenant, l'accès immédiat à l'information, la collaboration et surtout l'apprentissage à travers le temps et l'espace. Si nous revenons au modèle développé plus haut, nous pouvons confronter nos résultats avec ces principes théoriques.

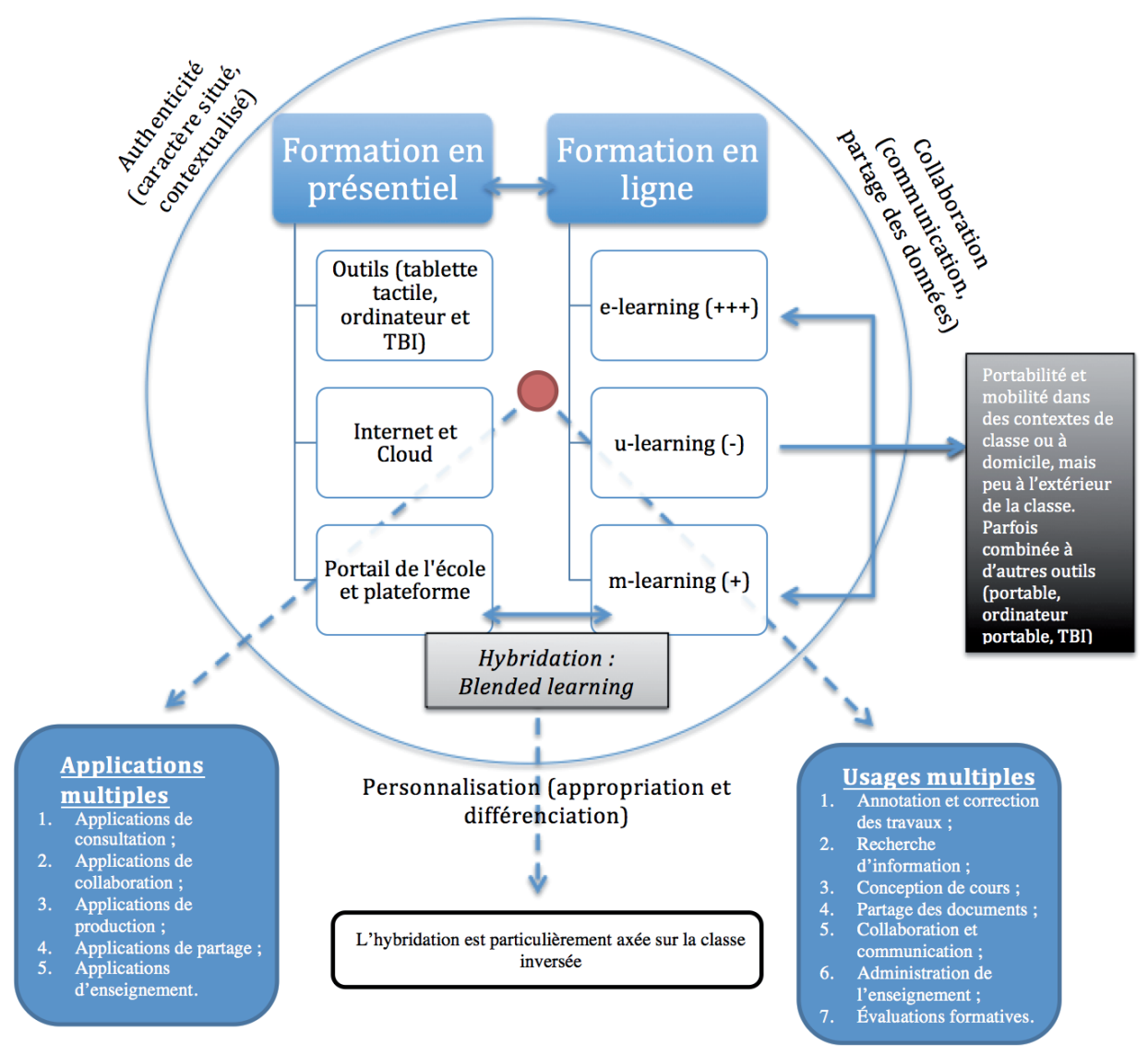

Figure 5

Modélisation des usages de la tablette en contexte scolaire (Fiévez, 2017). 
Nos résultats montrent clairement que la tablette prend une place centrale dans la salle de classe tant sur le plan de la formation en présentiel que sur celui de la formation en ligne. Nous pouvons également ajouter différents usages et applications au modèle théorique. Des usages comme l'annotation et la correction de travaux, la conception de cours, la réalisation d'évaluations peuvent être ajoutés. Des applications multiples, comme des applications de consultation, de production, de collaboration, de partage, mais également d'enseignement, sont présentes. L'enseignant utilise la tablette comme un outil complémentaire à l'enseignement et à l'apprentissage. Il fait évoluer la salle de classe vers d'autres perspectives pédagogiques, qui tendent vers l'ouverture de la classe sur le monde extérieur. L'enseignant, mais aussi les élèves, tend vers un apprentissage mobile, voire ubiquitaire. En effet, la tablette s'utilise en dehors de la salle de classe, comme à la maison ou lors des trajets. De fait, l'apprentissage ubiquitaire est également présent chez les enseignants interrogés. Ils combinent souvent, lors des activités d'enseignement et de conception, plusieurs outils technologiques, comme l'ordinateur ou le TBI. Cependant, force est de constater que le blended learning est peu utilisé. Les modèles précités par les auteurs dans la figure 1 sont peu usités et se limitent à la classe inversée. Ainsi, si globalement la tablette intègre un enseignement plus ouvert, mobile, collaboratif et hybride, il n'est pas encore suffisamment ouvert vers l'extérieur et vers un enseignement combinant l'apprentissage flexible et personnalisé où l'élève contribue significativement à son apprentissage, induisant concrètement ses besoins spécifiques. De fait, la personnalisation (autorégulation et choix de l'apprenant) envisagée dans le modèle théorique n'est pas très présente. Nous concluons que celui-ci doit être modifié en fonction des réalités du terrain. Enfin, même si les enseignants privilégient un contexte authentique, collaboratif et personnalisé, des ressources telles que la géolocalisation ou la réalité augmentée s'intègrent très peu dans la formation présentielle. Comme nous le constatons, l'utilisation de la tablette en salle de classe montre des usages pédagogiques intéressants, mais en attente d'innovation. La tablette est un outil récent et son intégration est souvent complexe. Pour ce faire, d'autres analyses sont nécessaires afin de conceptualiser et d'identifier les pratiques pédagogiques avec cet outil, mais aussi avec d'autres outils. En effet, en y regardant de plus près, ce sont les concepts d'intégration des TIC au sens large, de pratique professionnelle et d'innovation qui font écho aux usages pédagogiques novateurs observés dans les salles de classe (voir Fiévez, 2017).

\section{Références}

Alyahya, S. et Gall, J. E. (2012). iPads in education: A qualitative study of students' attitudes and experiences. Dans T. Amiel et B. Wilson (dir.), World Conference on Educational Multimedia, Hypermedia and Telecommunications 2012 (p. 1266-1271). Waynesville, NC : Association for the Advancement of Computing in Education.

Babnik, P., Dorfinger, J., Ebner, M., Meschede, K., Mulley, U. et Widmer, M. (2011). Tenhnologieeinsatz in der schule. Dans M. Ebner et S. Schön (dir.), Lehrbuch für lernen und lehren mit technologien. Repéré à http://13t.tugraz.at/index.php/LehrbuchEbner10/article/view/47

Bansavich, J. C. (2011). iPad study at USF. Repéré à http://studylib.net/doc/14583351/ipad-study-at-usf\#

Beauchamp, G., Burden, K. et Abbinett, E. (2014). Teachers learning to use the iPad in Scotland and Wales : A new model of professional development. Journal of Education for Teaching, 41(2), 161-179.

Beebe, A. (2011). iPads in the college composition classroom: A pilot program at the University of Texas at Tyler.

Benson, C. L. (2013). The iPad: Novelty or breakthrough for science education? (Thèse de maîtrise, Montana State University, Bozeman). Repéré à https://scholarworks.montana.edu/xmlui/handle/1/2757 
Bernet, E. et Karsenti, T. (2013). Modes d'intégration et usages des TIC au troisième cycle du primaire : une étude multicas. Éducation et francophonie, 41(1), 45-69. http://dx.doi.org/10.7202/1015059ar

Boéchat-Heer, S. (2014). Évaluation de l'intégration de tablettes numériques dans deux établissements du canton de Neuchâtel. Repéré à http://edudoc.ch/record/112518/files/RapportRechercheStephaniBoechatHeer site 140430.pdf

Bogdan, R. C. et Biklen, S. K. (2003). Research for education: An introduction to theories and methods (4éd.). Boston, MA : Allyn and Bacon.

Boudokhane, F. (2006). Comprendre le non-usage technique : réflexions théoriques. Les enjeux de l'information et de la communication, 2006(1), 13-22.

Bruce, F., Bourbous, V., El-Chami, M., Eliot, J. et Howard, S. (2012, juillet). iPads: Outreach, collaboration, and innovation in academic libraries. Communication présentée à la ALIA Biennial 2012 Conference, Sydney. Repéré à https://web.archive.org/web/20160315234926/http://conferences.alia.org.au/alia2012/Papers/39 Freya.Bruce.pdf

Bruner, J. (1985). Vygotski : A historical and conceptual perspective. Dans J. V. Wertsch (dir.), Culture, Communication, and Cognition: Vygotskian Perspectives (p. 21-34). Cambridge : Cambridge University Press.

Burden, K. et Maher, D. (2014). Mobile technologies and authentic learning in the primary school classroom. Dans S. Younie, M. Leask et K. Burden (dir.), Teaching with ICT in the primary school (2e éd., p. 171-183). Londres : Routledge.

Churchill, D. et Wang, T. (2014). Teacher's use of iPads in higher education. Educational Media International, 51(3), 214225. http://dx.doi.org/10.1080/09523987.2014.968444

David, B. T., Yin, C. et Chalon, R. (2007). Contextual mobile learning for appliance mastery. Proceedings of IADIS International Conference Mobile Learning 2007, <http://www. researchgate.net/publication/266456646_Contextual_ Mobile_Learning_for_Appliance_Mastery>, consulté le 23 août 2016.

Dépelteau, F. (2011). La démarche d'une recherche en sciences humaines : de la question de départ à la communication des résultats ( $2^{\mathrm{e}}$ éd.). Bruxelles : De Boeck.

Derobertmasure, A. et Robertson, J. E. (2013). Data analysis in the context of teacher training: code sequence analysis using QDA Miner. Quality E Quantity, 48(4), 2255-2276. http://dx.doi.org/10.1007/s11135-013-9890-9

Derycke, A. (2006, septembre). Du e-learning au pervasive-learning : concepts, exemples et questions de recherche. Communication présentée à UBIMOB’06, Paris. Repéré à http://www-inf.int-evry.fr/ defude/UbiMob06/DeryckeUBIMOB06.pdf

Fabian, K. et MacLean, D. (2014). Keep taking the tablets? Assessing the use of tablet devices in learning and teaching activities in the further education sector. Research in Learning Technology, 22. http://dx.doi.org/10.3402/rlt.v22.22648

Fernández-López, Á., Rodríguez-Fórtiz, M. J., Rodríguez-Almendros, M. L. et Martínez-Segura, M. J. (2013). Mobile learning technology based on iOS devices to support students with special education needs. Computers Education, 61, 77-90. http://dx.doi.org/10.1016/j.compedu.2012.09.014

Fielding, N. G. (2012). Triangulation and mixed methods designs: Data integration with new research technologies. Journal of Mixed Methods Research, 6(2), 124-136. http://dx.doi.org/10.1177/1558689812437101

Fiévez, A. (2017). L’intégration des TIC en contexte éducatif: modèles, réalités et enjeux. Québec, QC : Presses universitaires du Québec.

Flewitt, R., Kucirkova, N. et Messer, D. (2014). Touching the virtual, touching the real: iPads and enabling literacy for students experiencing disability. Australian Journal of Language and Literacy, 37(2), 107-116. Repéré à http://eprints.ncrm.ac.uk/3366/1/Flewitt Kucirkova and Messer 2014 Touching the virtual touching the real.pdf

Gesser, C. (2011). mLearning : Mobile devices as research and teaching tools [Présentation PowerPoint], <http://fr.slideshare. net/profgesser/mlearning-mobile-devices-as- research-and-teaching-tools>, consulté le 23 août 2016.

Gicquel, P.-Y. (2010). Vers une modélisation des situations d'apprentissage ubiquitaire. Dans V. Guéraud et M. Lefevre (dir.), Actes des troisièmes Rencontres Jeunes Chercheurs en EIAH (p. 93-98). Repéré à https://hal.archives-ouvertes.fr/hal-00506967 
Giroux, P., Coulombe, S., Cody, N. et Gaudreault, S. (2013). L'utilisation de tablettes numériques dans des classes de troisième secondaire : retombées, difficultés, exigences et besoins de formation émergents. STICEF, 20. Repéré à http://sticef.univ-lemans.fr/num/vol2013/07-giroux-cren/sticef 2013 NS giroux 07.htm

Henderson, S. et Yeow, J. (2012). iPad in education: A case study of iPad adoption and use in a primary school. Dans R. H. Sprague (dir.), 45 th Hawaii International Conference on System Science (p. 78-87). Repéré à http://www.computer.org/csdl/proceedings/hicss/2012/4525/00/4525a078.pdf

Huber, S. (2012). iPads in the classroom: a development of a taxonomy for the use of tablets in schools. Repéré à http://13t.eu/itug/images/band2.pdf

Hutchison, A. et Beschorner, B. (2014). Using the iPad as a tool to support literacy instruc- tion. Technology, Pedagogy and Education, 24(4), 407-422.

Hutchison, A., Beschorner, B. et Schmidt-Crawford, D. (2012). Exploring the use of the iPad for literacy learning. The Reading Teacher, 66(1), 15-23. http://dx.doi.org/10.1002/TRTR.01090

International Data Corporation. (2014, 30 octobre). Fueled by back-to-school promotions and US growth, the worldwide tablet market grows $11.5 \%$ in the third quarter, according to IDC. Repéré à https://web.archive.org/web/20141101234111/http://www.idc.com/getdoc.jsp? containerId=prUS25225114

Karsenti, T. et Collin, S. (2011). Une étude sur les apports des ordinateurs portables au primaire et au secondaire. Dans G.-L. Baron, E. Bruillard et V. Komis (dir.), Sciences et technologies de l'information et de la communication en milieu éducatif : analyse de pratiques et enjeux didactiques (p. 261-270). Repéré à https://halshs.archives-ouvertes.fr/edutice-00676148

Karsenti, T. et Fiévez, A. (2013). L'iPad à l'école : usages, avantages et défis : résultats d'une enquête auprès de 6057 élèves et 302 enseignants du Québec (Canada). Repéré à http://www.karsenti.ca/ipad/pdf/rapport iPad Karsenti-Fievez FR.pdf

Karsenti, T., Komis, V., Depover, C. et Collin, S. (2011). La recherche en éducation à l'ère des TIC. Dans T. Karsenti et L. Savoie-Zjac (dir.), La recherche en éducation : étapes et approches (3e éd., p. 168-192). Ville Saint-Laurent : ERPI.

Kearney, M. et Maher, D. (2013). Mobile learning in math teacher education : Using iPads to support pre-service teachers' professional development. Australian Educational Computing, 27(3), 76-84, <http://acce.edu.au/sites/acce.edu.au/ files/pj/journal/ AEC27-3_KearneyMaher.pdf>, consulté le 23 août 2016.

Kearney, M., Schuck, S., Burden, K. et Aubusson, P. (2012). Viewing mobile learning from a pedagogical perspective. Research in Learning Technology, 20. http://dx.doi.org/10.3402/rlt.v20i0/14406

Kinash, S., Brand, J. et Mathew, T. (2012). Challenging mobile learning discourse through research: Student perceptions of Blackboard Mobile Learn and iPads. Australian Journal of Educational Technology, 28(4), 639-655. http://dx.doi.org/10.14742/ajet.832

Kukulska-Hulme, A. et Traxler, J. (2005). Mobile learning: A handbook for educators and trainers. New York, NY : Routledge.

L'Écuyer, R. (1990). Méthodologie de l'analyse développementale de contenu. Méthode GPS et concept de soi. Sainte-Foy, QC : Presses de l'Université du Québec.

Laroussi, M. (2012, mai). Les nouvelles modalités d'apprentissage : e-learning, mobile learning, ubiquitous learning, pervasive learning, elearning 2.0, serious game. Communication présentée aux Premières journées doctorales en systèmes d'information, réseaux et télécommunication, Rabat. Repéré à http://ensias.um5s.ac.ma/jdsirt/JDISIRT15 files/ JDSIRT13/JDISIRT13 files/jdsirt12/InterventionMonaLaroussi.pdf

Lederman, N. G. et Abell, S. K. (2014). Handbook of research on science education. New York, NY : Routledge.

Liaw, S.-S., Hatala, M. et Huang, H.-M. (2010). Investigating acceptance toward mobile learning to assist individual knowledge management: based on activity theory approach. Computers E Education, 54(2), 446-454. http://dx.doi.org/10.1016/j.compedu.2009.08.029

Michel, C., Sandoz-Guermond, F. et Serna, A. (2012). Revue de littérature sur l'évaluation de l'usage de dispositifs mobiles et tactiles ludo-éducatifs pour les jeunes enfants. Dans Conférence EIAH 2011. Lyon : INSA-Lyon, <http://liris.cnrs. fr/Documents/Liris-5385. pdf>, consulté le 23 août 2016. 
Milot, V. (2010). Émergence d'un nouveau paradigme éducationnel : le mobile-learning: Évaluation de l'apport des technologies mobiles en contexte d'apprentissage. Editions universitaires européennes.

Mockus, L., Dawson, H., Edel-Malizia, S., Sha er, D. et Swaggerty, A. (2011). The Impact of Mobile Access on Motivation: Distance Education Student Perceptions, <http://learning- design.psu.edu/assets/uploads/pdf/MLRTWhitePaper.pdf>, consulté le 23 août 2016.

Montrieux, H., Vanderlinde, R., Courtois, C., Schellens, T. et De Marez, L. (2014). A qualitative study about the implementation of tablet computers in secondary education: the teachers' role in this process. Procedia - Social and Behavioral Sciences, 112, 481-488. http://dx.doi.org/10.1016/j.sbspro.2014.01.1192

Murray, O. T. et Olcese, N. R. (2011). Teaching and learning with iPads, ready or not? TechTrends, 55(6), 42-48. http:// dx.doi.org/10.1007/s11528-011-0540-6

Nincarean, D., Alia, M. B., Halim, N. D. A. et Rahman, M. H. A. (2013). Mobile augmented reality: The potential for education. Procedia - Social and Behavioral Sciences, 103, 657-664. http://dx.doi.org/10.1016/j.sbspro.2013.10.385

Northrop, L. et Killeen, E. (2013). A framework for using iPads to build early literacy skills. The Reading Teacher, 66(7), 531-537. http://dx.doi.org/10.1002/TRTR.1155

O’Malley, C., Vavoula, G., Glew, J. P., Taylor, J., Sharples, M., Lefrere, P., . . Waycott, J. (2005). Guidelines for learning/ teaching/tutoring in a mobile environment. Repéré à https://hal.archives-ouvertes.fr/hal-00696244

O'Sullivan, J. (2012). The teacher's ultimate app guide and resource book on how to use the iPad. Repéré à http://yourpadormine. weebly.com/uploads/1/8/5/3/1853836/ultimate teachers ipad guide.pdf

OCDE. (2015). Perspectives des politiques de l'éducation 2015 : les réformes en marche. Paris: OCDE. http://dx.doi.org/10.1787/9789264227330-fr

Ostler, E. et Topp, N. (2013). Digital note taking: An investigation of an iPad application as a strategy for content review and practice in intermediate algebra. Dans R. McBride et M. Searson (dir.), Proceedings of Society for Information Technology et Teacher Education International Conference 2013 (p. 72-77). Chesapeake, VA : Association for the Advancement of Computing in Education.

Paladino-Christin, M. (2015). Podcasting et autoformation : préférences d'usage et aspects motivationnels. Le cas des professionnels des technologies de l'information et de la communication (TIC) (Thèse de maîtrise, Université de Genève). Repéré à https://archive-ouverte.unige.ch/unige:55039

Park, Y. (2011). A pedagogical framework for mobile learning: Categorizing educational applications of mobile technologies into four types. International Review of Research in Open and Distance Learning, 12(2), 78-102. http://dx.doi.org/10.19173/irrodl.v12i2.791

Pellerin, G. (2015). Lagrange, J.-B. (dir.). (2013). Les technologies numériques pour l'enseignement. Usages, dispositifs et genèses. Toulouse : Octares. Formation et profession, 23(1), 92-94. http://dx.doi.org/10.18162/fp.2015.a56

Peluso, D. C. C. (2012). The fast-paced iPad revolution: Can educators stay up to date and relevant about these ubiquitous devices? British Journal of Educational Technology, 43(4), E125-E127. http://dx.doi.org/10.1111/j.1467-8535.2012.01310.x

Penny, C., Shugar, J., McConatha, D., Bolton, D. et Taylor, P. (2013). The higher education classroom in the post PC era. Dans R. McBride et M. Searson (dir.), Proceedings of Society for Information Technology E Teacher Education International Conference 2013 (p. 3760-3762). Repéré à https://www.learntechlib.org/p/48695

Pintrich, P. R. et Schunk, D. H. (2002). Motivation in education: Theory, research, and applications ( ${ }^{\mathrm{e}}$ éd.). Upper Saddle River, NJ : Merrill Prentice-Hall.

Portney, L. G. et Watkins, M. P. (2009). Foundations of Clinical Research : Applications to Practice. Upper Saddle River, NJ : Pearson/Prentice Hall.

Proulx, J. (2004). L'apprentissage par projet. Québec, QC : Presses de l'Université du Québec.

Proulx, S. et Breton, P. (2002). L’explosion de la communication à l'aube du XXI' siècle. Montréal, QC : Boréal. 
Raby, C. (2004). Analyse du cheminement qui a mené des enseignants du primaire à développer une utilisation exemplaire des TIC en classe (Thèse de doctorat, Université du Québec à Montréal). Repéré à https://halshs.archives-ouvertes.fr/edutice-00000750

Raby, C., Karsenti, T., Meunier, H. et Villeneuve, S. (2011). Usage des TIC en pédagogie universitaire : point de vue des étudiants. Revue internationale des technologies en pédagogie universitaire, 8(3), 6-19. http://dx.doi.org/10.7202/1006396ar

Rhodes, J. A. (2013). Exploring writing with iPads: instructional change for pre-service educators. Dans K. E. Pytash, R. E. Ferdig et T. V. Rasinki (dir.), Preparing teachers to teach writing using technology (p. 57-68). Pittsburgh, PA : ETC Press.

Royer, C., Baribeau, C. et Duchesne, A. (2009). Les entretiens individuels dans la recherche en sciences sociales au Québec : où en sommes-nous? Un panorama des usages. Recherches qualitatives, HS7, 64-79, <http://www.recherchequalitative.qc.ca/docu- ments/ les/revue/hors_serie/hors_serie_v7/HS7_Texte_Royer_Baribeau.pdf>, consulté le 23 août 2016.

Shah, N. (2011). Special education pupils find learning tool in iPad applications. Education Week, 30(22), 1-17. Repéré à http://www.edweek.org/ew/articles/2011/03/02/22ipad.h30.html

Sockett, G. et Kusyk, M. (2013). L'apprentissage informel en ligne : nouvelle donne pour l'en- seignement-apprentissage de l'anglais. Recherche et pratiques pédagogiques en langues de spécialité. Cabiers de l'Apliut, 12(1), 75-91, <http://doi. org/10.4000/apliut.3578>, consulté le 23 août 2016.

Staker, H. et Horn, M. B. (2012). Classifying K-12 blended learning. Repéré à http://eric.ed.gov/?id=ED535180

Tondeur, J., van Braak, J., Sang, G., Voogt, J., Fisser, P. et Ottenbreit-Leftwich, A. (2012). Preparing pre-service teachers to integrate technology in education: A synthesis of qualitative evidence. Computers E Education, 59(1), 134-144. http://dx.doi.org/10.1016/j.compedu.2011.10.009

Underwood, J., et Dillon, G. (2011). Chasing dreams and recognising realities: teachers' responses to ICT. Technology, Pedagogy and Education, 20(3), 317-330. http://dx.doi.org/10.1080/1475939x.2011.610932

Van der Maren, J.-M. (1996). Méthodes de recherche pour l'éducation (2e éd). Bruxelles : De Boeck Supérieur.

Villemonteix, F., Hamon, D., Nogry, S., Séjourné, A., Hubert, B. et Gélis, J.-M. (2014). Expérience tablettes tactiles à l'école primaire - ExTaTE. Repéré à https://hal.archives-ouvertes.fr/hal-01026077v2

Vu, P. H. (2013). An inquiry into how iPads are used in classrooms (Dissertation). Repéré à http://opensiuc.lib.siu.edu/cgi/viewcontent.cgi?article=1708\&context=dissertations

Walters, E. A. et Baum, M. (2011). Will the iPad revolutionize education ? Learning E Leading with Technology, 38(7), 6-7.

Wishard, N. L. (2015). All iPads, all the time: A qualitative study of high school teachers' experiences with and perspectives on the use of exclusive technology (Thèse de doctorat). Accessible par ProQuest Dissertations \& Theses. (3687325)

\section{Pour citer cet article}

Fiévez, A. et Karsenti, T. (2018). Usages et perceptions des enseignants lors de l'utilisation de la tablette en contexte scolaire. Formation et profession, 26(1), 55-73. http://dx.doi.org/10.18162/fp.2018.394 